\title{
Exoplanet imaging performance envelopes for starshade-based missions (Erratum)
}

\author{
Eliad Peretz, ${ }^{\text {a,* }}$ Kevin Hall, ${ }^{\text {b }}$ John C. Mather, ${ }^{\text {a }}$ Stuart Shaklan, \\ and Sergi Hildebrand ${ }^{\mathrm{c}}$ \\ ${ }^{a}$ NASA Goddard Space Flight Center, Greenbelt, Maryland, United States \\ ${ }^{b}$ University of Maryland, College Park, Department of Astronomy, College Park, \\ Maryland, United States \\ ${ }^{\mathrm{c}}$ Jet Propulsion Laboratory, California Institute of Technology, Pasadena, California, \\ United States
}

[DOI: 10.1117/1.JATIS.7.2.029801]

This article [J. Astron. Telesc. Instrum. Syst. 7(2), 021215 (22 March 2021) https://doi.org/10 .1117/1.JATIS.7.2.021215] was originally published with Figs. 2 and 3 reversed. The figures have been put into their correct places in the paper.

All versions of the article were corrected on 7 April 2021.

*Address all correspondence to Eliad Peretz, eliad.peretz@nasa.gov 